\title{
Correlations between Motor Symptoms across Different Motor Tasks, Quantified via Random Forest Feature Classification in Parkinson's Disease
}

\author{
Andreas Kuhner ${ }^{1,2}$, Tobias Schubert ${ }^{1,2}$, Massimo Cenciarini2,3,4, \\ Isabella Katharina Wiesmeier ${ }^{2,3,4}$, Volker Arnd Coenen ${ }^{2,4,5}$, Wolfram Burgard ${ }^{1,2}$, \\ Cornelius Weiller ${ }^{2,3,4}$ and Christoph Maurer M $^{2,3,4 *}$
}

${ }^{1}$ Department of Computer Science, University of Freiburg, Freiburg, Germany, ${ }^{2}$ BrainLinks BrainTools, Cluster of Excellence, University of Freiburg, Freiburg, Germany, ${ }^{3}$ Department of Neurology and Neuroscience, Medical Center, University of Freiburg, Freiburg, Germany, ${ }^{4}$ Medical Faculty, University of Freiburg, Freiburg, Germany, ${ }^{5}$ Department of Stereotactic and Functional Neurosurgery, Medical Center, University of Freiburg, Freiburg, Germany

OPEN ACCESS

Edited by:

Maurizio Ferrarin,

Fondazione Don Carlo Gnocchi

Onlus (IRCCS), Italy

Reviewed by:

Jeffrey M. Hausdorff,

Tel Aviv University, Israel

Andrea Mannini,

Sant'Anna School of Advanced

Studies, Italy

*Correspondence:

Christoph Maurer

christoph.maurer@

uniklinik-freiburg.de

Specialty section:

This article was submitted

to Movement Disorders,

a section of the journal

Frontiers in Neurology

Received: 04 July 2017 Accepted: 31 October 2017

Published: 14 November 2017

Citation:

Kuhner A, Schubert T, Cenciarini M,

Wiesmeier IK, Coenen VA,

Burgard W, Weiller $C$ and Maurer $C$

(2017) Correlations between Motor

Symptoms across Different Motor

Tasks, Quantified via Random

Forest Feature Classification

in Parkinson's Disease.

Front. Neurol. 8:607.

doi: 10.3389/fneur.2017.00607
Background: Objective assessments of Parkinson's disease (PD) patients' motor state using motion capture techniques are still rarely used in clinical practice, even though they may improve clinical management. One major obstacle relates to the large dimensionality of motor abnormalities in PD. We aimed to extract global motor performance measures covering different everyday motor tasks, as a function of a clinical intervention, i.e., deep brain stimulation (DBS) of the subthalamic nucleus.

Methods: We followed a data-driven, machine-learning approach and propose performance measures that employ Random Forests with probability distributions. We applied this method to 14 PD patients with DBS switched-off or -on, and 26 healthy control subjects performing the Timed Up and Go Test (TUG), the Functional Reach Test (FRT), a hand coordination task, walking $10-\mathrm{m}$ straight, and a $90^{\circ}$ curve.

Results: For each motor task, a Random Forest identified a specific set of metrics that optimally separated PD off DBS from healthy subjects. We noted the highest accuracy (94.6\%) for standing up. This corresponded to a sensitivity of $91.5 \%$ to detect a PD patient off DBS, and a specificity of $97.2 \%$ representing the rate of correctly identified healthy subjects. We then calculated performance measures based on these sets of metrics and applied those results to characterize symptom severity in different motor tasks. Task-specific symptom severity measures correlated significantly with each other and with the Unified Parkinson's Disease Rating Scale (UPDRS, part III, correlation of $r^{2}=0.79$ ). Agreement rates between different measures ranged from 79.8 to $89.3 \%$.

Conclusion: The close correlation of PD patients' various motor abnormalities quantified by different, task-specific severity measures suggests that these abnormalities are only facets of the underlying one-dimensional severity of motor deficits. The identification and characterization of this underlying motor deficit may help to optimize therapeutic interventions, e.g., to "automatically" adapt DBS settings in PD patients.

Keywords: Parkinson's disease, motion, deep brain stimulation, random forest, sensor suit 


\section{INTRODUCTION}

The most frequently used tool for clinically assessing Parkinson's disease (PD) is the revised version of the Unified Parkinson's Disease Rating Scale [International Parkinson and Movement Disorder Society (MDS)-UPDRS, 1]. The Unified Parkinson's Disease Rating Scale (UPDRS) combines numerous clinical scales and questionnaires regularly used in clinical routine and in studies to evaluate the presence, severity, and progression of PD symptoms (1). However, the disadvantages of the UPDRS are that it is very time-consuming, its inter- and intra-rater variability, dependence on location, limitations involving regularly repeating the assessments, and the lack of quantitative outcomes (2). Adjusting deep brain stimulation (DBS) parameters in PD patients still relies on subjective estimations of patients' clinical state, despite the vast motor symptoms and adjustable parameters.

Motion capture techniques were recently introduced to characterize motor behavior in PD more objectively (2-4). Early attempts focused on evaluating individual symptoms $(5,6)$, using either single (7-9) or multiple-sensor systems (6,10-13). Overall, assessing motor symptoms using motion capture techniques seemed to correlate well with specific clinical tests for motor symptoms, such as tremor, bradykinesia, or dyskinesia (14). Since these assessments are usually closely related to the specific clinical tests, less is known about how these test results relate to more everyday life motor activities, such as walking, bending over, goal-directed hand movements, etc. Moreover, we do not yet know how these symptoms correlate with each other in the individual patient.

One of the benefits of motion capture techniques is that machine-learning approaches can be implemented to handle the growing amount of data (15). Some authors applied machine-learning approaches to automatically differentiate PD patients from healthy controls or to classify levels of motor impairment using various methods and parameters $[(16,17)$ : gait parameters, (18): quantitative EEG, (19): computer vision techniques]. Recently, Bernad-Elazari et al. (20) examined the benefit of a single body-fixed sensor to discriminate between PD patients and healthy controls. Machine-learning approaches have also been investigated to monitor tremor, dyskinesia, or bradykinesia in PD patients $(21,22)$. However, we are unaware of any study taking machine-learning approaches to derive a continuous measure of motor performance across many different motor tasks.

Earlier studies already aimed to evaluate motor abnormalities that directly target certain motor tasks, e.g., free stance, as the accurate identification of balance abnormalities is of high clinical relevance in $\operatorname{PD}(23,24)$. Even a seemingly simple task like the real-world detection of PD patients' falls is technically challenging. Numerous algorithms, devices, and device locations [chest, waist, shin, or wrist $(25,26)]$ have been tested to improve the accuracy of fall detection in PD patients. Still, these studies reveal significant variability in the outcomes they measured.

Objective assessments of PD patients' gait abnormalities may help the clinician to evaluate the efficacy of therapeutic interventions and are potentially useful as biological markers for PD's diagnosis, prognosis, and progression (27-29). The validities of various algorithms that detect discrete gait characteristics in the laboratory have already been investigated $(14,30-34)$. However, how these measures relate to each other and to other motor abnormalities in PD patients remains open to debate.

More general clinical tests of functional mobility such as the Timed Up and Go Test [TUG; (35-37)] combine movements that invariably incorporate postural transitions. Mean turn velocity, slower walking and turning, shorter steps, and lower cadence distinguished PD from controls $(35,37)$ and also revealed greater sensitivity to dysfunction than most other clinical rating scores $(35,38)$. Again, it remains unclear how closely the Timed Up and Go elements correlate with each other and with other motor abnormalities in the individual PD patient.

In this study, we aimed to detect abnormal motor behavior of PD patients performing several different motor tasks in two clinical conditions (DBS switched-off, -on). We extracted metric values from motion capture suit data and demonstrated that each performed task is best characterized by a specific metric. Then, we combined all metrics with a Random Forest approach and calculated the most discriminative markers for abnormality in each motor task. These Random Forests, enhanced by probability distributions, were then used as symptom severity measures. Finally, we evaluated how well these task-specific severity measures correlated with each other.

\section{MATERIALS AND METHODS}

\section{Subjects and Data}

We used the XSens motion capture suit to record the motion data of 26 healthy subjects [ 11 females, 15 males, mean age $54.7 \pm 8.8$ years $( \pm$ SD) $]$ and 14 PD patients ( 5 females, 9 males, mean age $59.1 \pm 11.1$ years, for detailed information, see Table 1 ). The motion capture suit consists of 17 MEMS (microelectromechanical systems) with outputs based on the fusion of signals stemming from 3D inertial measurement units (IMUs), ie, linear accelerometers, 3D magnetometers, and 3D rate gyroscopes. This system's raw outcome is velocity traces. It records data with a frame rate of $120 \mathrm{~Hz}$ and stores positional and rotational data of 23 body parts (Figure 1).

Parkinson's disease patients were recruited during the initial programming for bilateral DBS of the subthalamic nucleus. Stimulator implantation followed established guidelines. The study protocol involved the Timed Up and Go Test (TUG), the Functional Reach Test (FRT), a 10-m Walk Test (39), a $90^{\circ}$ turn to right and to the left, and a hand coordination task (pouring water from a glass in the right hand to a glass in the left hand and vice versa). The TUG and the FRT were repeated three times. Our analysis was based on 20 to 30 steps during straight walking, two $90^{\circ}$ turns for each direction, and 12 hand coordination tasks. PD patients were recorded in both the stimulation switchedoff (off DBS) and switched-on (on DBS) condition while taking their regular L-Dopa medication. The levodopa-equivalent doses were calculated according to Ref. (40). 
TABLE 1 | Data of patients with Parkinson's disease.

\begin{tabular}{|c|c|c|c|c|c|c|c|}
\hline \multirow[t]{2}{*}{ Patient } & \multirow[t]{2}{*}{ Gender } & \multirow[t]{2}{*}{$\begin{array}{c}\text { Age } \\
\text { (years) }\end{array}$} & \multirow{2}{*}{$\begin{array}{c}\text { Disease } \\
\text { duration } \\
\text { (years) }\end{array}$} & \multirow{2}{*}{$\begin{array}{l}\text { Time since } \\
\text { Stim. Impl. } \\
\text { (months) }\end{array}$} & \multirow{2}{*}{$\begin{array}{l}\text { LEDD } \\
\text { (mg) }\end{array}$} & \multicolumn{2}{|c|}{$\begin{array}{l}\text { UPDRS motor } \\
\text { score }\end{array}$} \\
\hline & & & & & & DBS off & DBS on \\
\hline 1 & $F$ & 74 & 11 & 1 & 0 & 20 & 15 \\
\hline 2 & $\mathrm{~F}$ & 69 & 8 & 1 & 0 & 29 & 20 \\
\hline 3 & $\mathrm{M}$ & 74 & 12 & 1 & 607 & 43 & 22 \\
\hline 4 & $\mathrm{~F}$ & 49 & 9 & 1 & 475 & 51 & 32 \\
\hline 5 & $\mathrm{~F}$ & 53 & 11 & 1.5 & 700 & 38 & 29 \\
\hline 6 & $\mathrm{M}$ & 57 & 14 & 1 & 349.5 & 47 & 15 \\
\hline 7 & $M$ & 40 & 10 & 1 & 475 & 61 & 16 \\
\hline 8 & $M$ & 43 & 10 & 2 & 1,308.3 & 28 & 15 \\
\hline 9 & $M$ & 66 & 21 & 1 & 717.5 & 42 & 35 \\
\hline 10 & $M$ & 55 & 25 & 1 & 0 & 43 & 36 \\
\hline 11 & $\mathrm{~F}$ & 61 & 14 & 1.5 & 525.75 & 34 & 12 \\
\hline 12 & $\mathrm{M}$ & 72 & 3 & 1.5 & 0 & 36 & 20 \\
\hline 13 & $M$ & 52 & 14 & 2 & 0 & 21 & 22 \\
\hline 14 & $M$ & 62 & 26 & 1.5 & 379.25 & 36 & 16 \\
\hline Mean & & 59.1 & 13.4 & 1.3 & 395.5 & 37.8 & 21.8 \\
\hline SD & & 11.1 & 6.4 & 0.4 & 367.30 & 11.3 & 8.0 \\
\hline
\end{tabular}

F, female, M, male, Stim. Impl., stimulator implantation, UPDRS, Unified Parkinson's Disease Rating Scale, DBS, deep brain stimulation, $L E D D$, levodopa-equivalent doses.

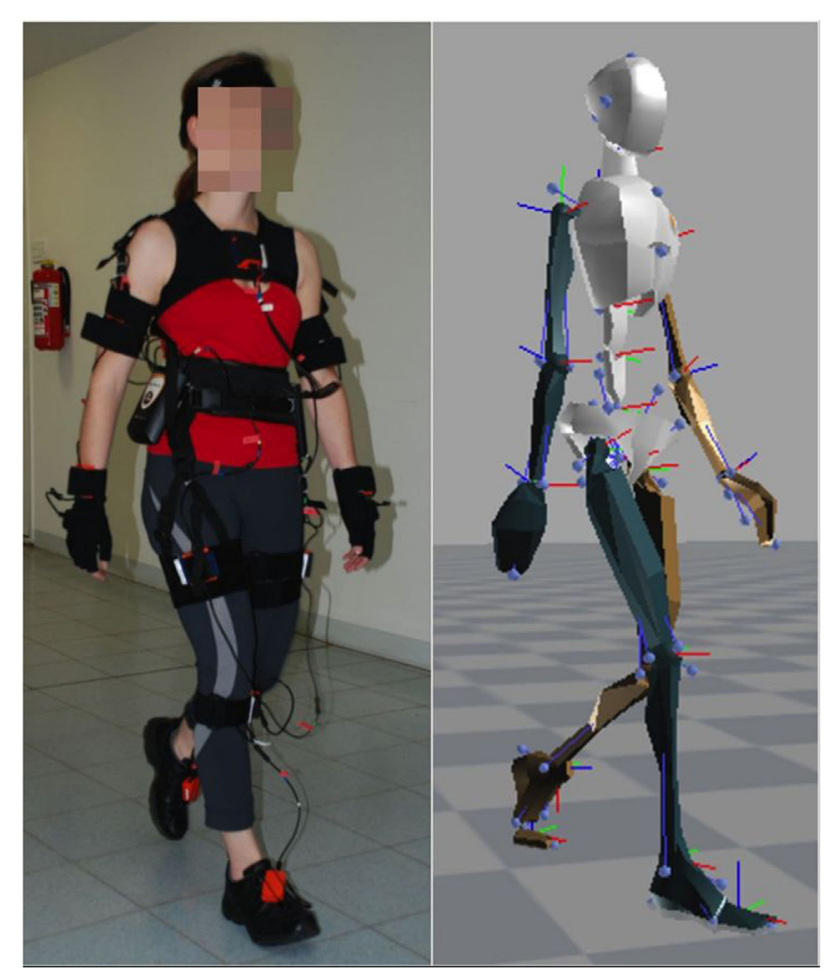

FIGURE 1 | XSens motion capture suit and corresponding avatar.

In addition, patients were assessed with the Unified Parkinson's Disease Rating Scale [International Parkinson and MDS-(part III), 1]. The UPDRS motor exam (part III) represents the patients' state by evaluating multiple motions like walking and hand coordination. Additionally, the UPDRS (part III) rates the stiffness of limbs or the ability to regularly perform repeated movements.

All subjects and patients gave their written informed consent and their data were included anonymized according to the Declaration of Helsinki. We carried out this study in accordance with the guidelines of the Ethics Committee of the Medical Center, University of Freiburg and national guidelines. The Ethics Committee of the Medical Center, University of Freiburg approved the study protocol.

\section{Metrics}

To calculate our metrics, all motor tasks except FRT were segmented into simpler elements, e.g., single steps, single hand movements for pouring water, or sub-tasks of the TUG like standup, turn, and sit down.

For these individual elements of tasks we use velocity time series from each body segment to derive single metrics. The Joint Activity (JA, dimensionless) represents the distance between an individual's motion velocity pattern and the average patterns of healthy subjects. It covers all metrics based on velocity traces and yields the degree of abnormality in terms of deviation from the normal range. This deviation represents a single segment velocity trace's different timing and movement profile during a sub-task. The distance is the probability that a given velocity pattern will match the average motion pattern of healthy subjects (41). As a single velocity trace could permanently remain within the range of normal behavior and still be highly abnormal due to, for example, high-frequency oscillations, we extended the metric "Joint Activity" by a set of smoothness- and frequency-related metrics. Our metrics describing the trajectory's smoothness included the normalized mean absolute jerk [NMAJ $\left.\left(1 / \mathrm{s}^{2}\right)\right]$, dimensionless jerk [DJ (dimensionless)], log dimensionless jerk [LDJ (dimensionless)], root mean square jerk $\left[\mathrm{RMSJ}\left(\mathrm{m} / \mathrm{s}^{3}\right)\right]$, and speed arc length [SpAL (dimensionless) (42)]. For details concerning the calculation of these metrics, see Supplementary Material. A metric representing the frequency content of a movement was spectral arc length [SAL (dimensionless)].

We apply the set of metrics to each of an individual's various body parts. Here, a sample consists of the 23 body part velocity profiles from a subject in one of the sub-tasks, e.g., a single step. Overall, each sample yields a 161 dimensional metric vector, in other words 7 metrics for 23 body parts.

\section{Random Forests with Probability Distributions}

Due to the high-dimensional data vectors, it is difficult to identify the most useful features for characterizing pathological movements. To overcome this challenge, we compute for each subtask a Random Forest (43) which combines the aforementioned metric vectors and filters the highly dimensional data to compute classification and performance measures. Our Random Forest approach consists of multiple decision trees. In the learning phase we optimize each decision tree on a subset of the training samples with a subset of metrics, i.e., we use $40 \%$ randomly chosen samples, i.e., task elements, from the training set and 11 randomly 
chosen metrics for each tree. Each decision tree is composed of nodes in which we split the training data into two subsets. Each node separates the training data through a randomly chosen threshold on a single metric. From all the decision tree's metrics, we choose the one that provides the most information. We repeat this procedure from node to node as long as we have a nonunique data set or reach a certain depth.

The Random Forest calculates the class of a new sample by supplying each decision tree with the sample data to obtain a single outcome. Furthermore, the Random Forest applies a majority vote over all decision trees to represent the final result. This leads to a binary decision.

We trained the algorithms to create a contrast between healthy and maximum pathological state, e.g., PD off DBS. The purpose of this binary decision was to apply it as a quality measure to compare different metrics and combinations of metrics derived from Random Forests on the basis of their accuracy, that is, their recognition rate to identify the correct subject group (see Table 2).

\section{Symptom Severity Measure}

We then go one step further and compute continuous performance measures in the interval $[0,1]$. In so doing, we extend each node via a probability distribution over the nodes' specific subset of training data and, while supplying the nodes of the decision tree with a new sample, multiply the sample's probabilities in the node's particular distribution. We obtain a sample data point's performance measure by summing up each decision tree's probabilities and normalizing the result. The purpose of this performance measure was to measure symptom severity.

More specifically, the Random Forests' training was based on a data set that represents both extremes of a disease: healthy subjects and PD patients off DBS. This provides us with a contrast to create a measure that describes all states in between. The Random Forest itself "learns" an exclusively either-or classification, while our performance measure utilizes the underlying tree structure to calculate a real-valued number between 0 and 1 . Furthermore, the composition of decision trees that are trained on a reduced training set size and with a subset of metric values

TABLE 2 | Accuracies for each executed task over all metrics.

\begin{tabular}{lcccccccc}
\hline & JA & DJ & LDJ & NMAJ & RMSJ & SAL & SpAL & Combined \\
\hline Steps & $\mathbf{8 5 . 7}$ & 59.6 & 60.1 & 57.0 & 80.8 & $\mathbf{8 8 . 7}$ & 62.4 & $\mathbf{8 9 . 2}$ \\
Curve & 80.0 & 91.0 & $\mathbf{9 2 . 0}$ & 67.0 & 79.0 & 77.0 & $\mathbf{9 4 . 0}$ & $\mathbf{9 2 . 0}$ \\
Functional & 66.0 & 73.0 & 73.4 & $\mathbf{8 6 . 9}$ & 72.6 & 75.2 & $\mathbf{7 7 . 8}$ & $\mathbf{8 6 . 0}$ \\
reach & & & & & & & & \\
Hand task & 69.2 & 82.6 & 82.4 & 75.2 & 74.5 & $\mathbf{8 3 . 1}$ & $\mathbf{8 4 . 6}$ & $\mathbf{8 7 . 5}$ \\
Stand-up & 77.8 & 79.1 & 77.1 & 66.4 & $\mathbf{8 7 . 9}$ & 69.1 & $\mathbf{8 6 . 5}$ & $\mathbf{9 4 . 6}$ \\
Turn & 75.1 & 74.4 & 77.1 & 63.7 & $\mathbf{8 3 . 8}$ & 71.1 & $\mathbf{8 4 . 5}$ & $\mathbf{8 9 . 9}$ \\
Sit down & $\mathbf{8 7 . 9}$ & 81.8 & 83.2 & 82.5 & 79.8 & 67.7 & $\mathbf{8 4 . 5}$ & $\mathbf{8 8 . 5}$ \\
\hline
\end{tabular}

The best metrics for each task are highlighted. Accuracies refer to Parkinson's disease patients with deep brain stimulation switched-off compared to healthy control subjects. The values result from $7+1$ cross-validation tests.

$J A$, joint activity (dimensionless); DJ, dimensionless jerk; LDJ, Log dimensionless jerk;

NMAJ, normalized mean absolute jerk $\left(1 / \mathrm{s}^{2}\right)$; RMSJ, root mean square jerk $\left(\mathrm{m} / \mathrm{s}^{3}\right)$; $S A L$, spectral arc length $\left(1 / s^{3}\right)$, SpAL, speed arc length (dimensionless). enhances the generalization capabilities and prevents overfitting. We also carry out a leave-one-subject-out cross-validation. Hence, we remove one subject from the training set, teach our Random Forest on the training set and test its performance on the missing data sample. This procedure is repeated for each subject, and the resulting values depict our algorithm's performance.

One of the upsides of a Random Forest is its ability to detect high-level correlations between different metrics. Through the nested transitioning over many layers in a tree, one obtains a multidimensional threshold function that sets metrics in relation to each other. Hereby, PD motor abnormalities like asymmetric motor behavior are automatically detected and used if they carry additional information.

\section{Correlation Analysis}

We compared our results of combined metrics with the UPDRS (part III) in order to verify their reliability. The correlation of performance measures with each other and the UPDRS (part III) were analyzed via the Pearson correlation test.

\section{RESULTS}

\section{Classification Accuracy for Different Tasks}

Table 2 illustrates the results of all $7+1$ cross-validation tests of several tasks with different metrics and the combination of all metrics in one Random Forest between patients with off DBS and healthy controls. The seven tasks and sub-tasks are characterized by different sets of metrics indicating diverse motor abnormalities. In general, SpAL yielded high accuracy for all tasks except walking (steps). Specifically, walking a $90^{\circ}$ curve was best characterized by SpAL (94\%) and Log Dimensionless Jerk (LDJ, 92\%), whereas turning was best characterized by SpAL (84.5\%) and RMSJ (83.8\%). SAL and JA displayed the highest accuracy during walking (steps, 88.7 and $85.7 \%$, respectively). The highest accuracy regarding the FRT was achieved by SpAL (77.8\%) and NMAJ (86.9\%), whereas the Hand Coordination Task's highest accuracy was achieved by SpAL (84.6\%) and SAL $(83.1 \%)$. Standing up and sitting down were best characterized by SpAL (Stand-up: $86.5 \%$, sit down $84.5 \%$ ), RMSJ (Stand-up, $87.9 \%$ ), and JA, respectively (Sit down, 87.9\%). The agreement rate between different task-specific measures ranged from 79.8 to $89.3 \%$ with the highest agreement rate between standing up and the 10-m Walk Test.

We computed combined measures for each task using the Random Forest. The combined measures revealed similarly high accuracy over all tasks and sub-tasks. The highest combined accuracy (94.6\%) was achieved for the task "standing up." This corresponded to a sensitivity of $91.5 \%$ to detect a PD patient off DBS, and a specificity of $97.2 \%$ representing the rate of correctly identified healthy subjects.

\section{Symptom Severity Measures}

Using the decision tree's probabilities, we calculated performance measures that represent the severity of symptoms based on the average of likelihoods from each decision tree to be a PD patient off DBS. When applying the performance measure 
on PD patients on DBS, we observed an average value of 53.3\% - halfway between healthy and PD off DBS, as expected. Comparison of these combined performance measures with the UPDRS (part III) showed that their outcomes are similar (Figure 2). In all combined performance measures as well as in the UPDRS (part III), healthy control subjects scored low, followed by PD patients on DBS. PD patients off DBS got the highest scores.

\section{Improvement through DBS}

Figure 3 shows the combined performance measures of various tasks performed with DBS switched-off or on. Each PD patient (p01-p14) is depicted with a particular symbol and color. Most PD patients show lower performance measure values on DBS across all tasks. Note that the severity of motor symptoms and the effect of DBS in a single patient tended to be similar across different tasks.

\section{Correlation Analysis}

Correlating single metrics across different motor tasks leads to certain groups of motor tasks that correlate closely with each other. For example, the JA metrics derived from certain motor tasks (stand-up, turn, sit down, curve, and steps) correlated closely, while JA metrics derived from the hand coordination task or from functional reach did not correlate significantly. No single metric correlated significantly across all motor tasks.

However, if we take from each motor task the single best metric (apart from the combined, see Table 2), all different, task-specific metrics correlated significantly with each other (all $p$ values $<0.004)$. When correlating individual combinations

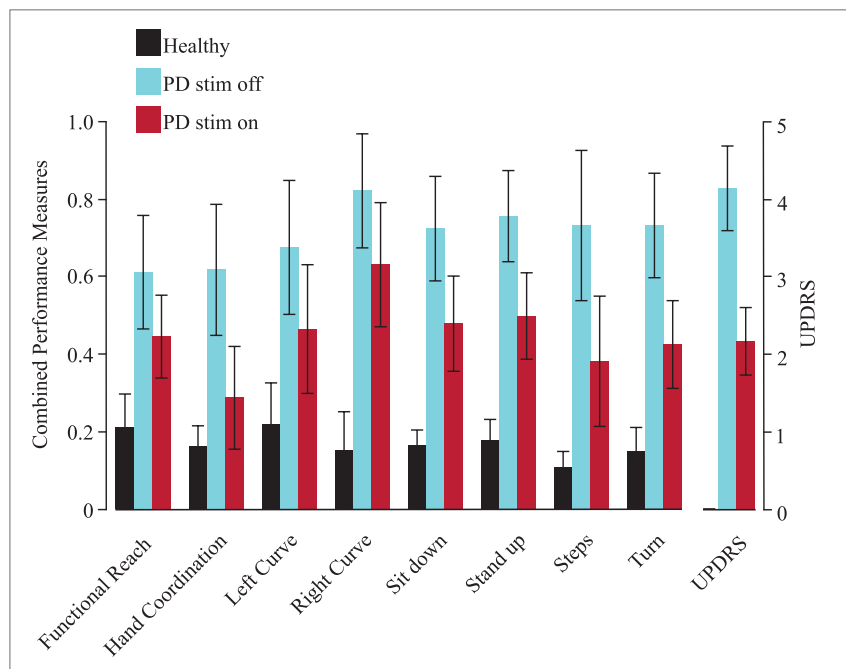

FIGURE 2 | Combined performance measures of all subjects for each task. The Unified Parkinson's Disease Rating Scale [Unified Parkinson's Disease Rating Scale (UPDRS), part III, right] is shown as a verifiability measure. Black represents healthy subjects, blue patients with deep brain stimulation (DBS) switched-off and red with DBS switched on. Error bars refer to the SE. PD, Parkinson's disease (patients), stim off, DBS switched-off, stim on, DBS switched on. of metrics (symptom severity measures) from each motor task, the correlations across all motor tasks were even higher ( $p$ values $<0.0003$ ). Moreover, task-specific combinations of severity measures correlated significantly with the UPDRS (part III). The first principal component alone represents $72 \%$ of the total variance of the data field and correlated significantly with the UPDRS (part III; $r^{2}=0.79, p<0.0001$, see Figure 4).

\section{DISCUSSION}

In this investigation, we took a data-driven approach aiming to identify symptom severity measures to comprehensively characterize motor abnormalities in PD. We examined motor behavior during several different everyday motor tasks [sit-to-stand, turning, stand-to-sit (Timed Up and Go Test, TUG), walking straight, walking a $90^{\circ}$ curve, leaning forward as much as possible (FRT), and a hand coordination task]. We selected these motor tasks specifically to represent the most relevant impairments in PD patients' quality of life $(2,14)$.

Data were recorded with a motion capture suit comprising 17 MEMS (microelectromechanical systems) with outputs based on the fusion of signals stemming from IMUs, magnetometers, and gyroscopes. Velocity traces were the raw outcome of this system. We used the data of all 17 MEMS for further analysis, as we did not want to bias the purely data-driven approach by choosing or excluding any segment arbitrarily. The Xsens sensor suit used here consisted of straps rather than a "suit," was "light weighted," and did not hamper any movement.

The minimum number of sensors required to capture reliable whole-body motion data are still unknown. Most investigators have applied few sensors to characterize single body parts [e.g., Ref. (44)]. Semwal et al. (45) used eight sensors to capture fullbody movements, but enriched sensor data with information on natural body posture. Future studies are needed to clarify this issue.

One limitation of this study is our relatively low number of healthy subjects $(n=26)$ and PD patients $(n=14)$ that could restrict our findings' generalizability. However, our approach was designed to identify severity measures of different motor symptoms in different motor tasks in individual PD patients. Moreover, the findings reported herein are based on a substantial amount of data, i.e., 23 body-part velocity profiles for a single element in a task (e.g., one step) and a 161 dimensional metric vector. Another limitation is that our metrics are based on segment velocity traces and we consequently miss the information from the initial positional offset in all joints. However, all other displacement-related abnormalities should be captured by these joint velocity traces.

Since PD patients' intended movement amplitudes or velocities are not known prior to the movement, we came up with a new metric named JA, which is based on the likelihood of a single joint velocity trace falling outside the normal range (41). JA may be abnormal even when the data distribution in healthy subjects is more complex, such as when it is bimodal. Theoretically speaking, a behavior can be considered abnormal if it is halfway between two normal ranges. In that respect, we believe that JA is a more broadly applicable measure for abnormality than too 


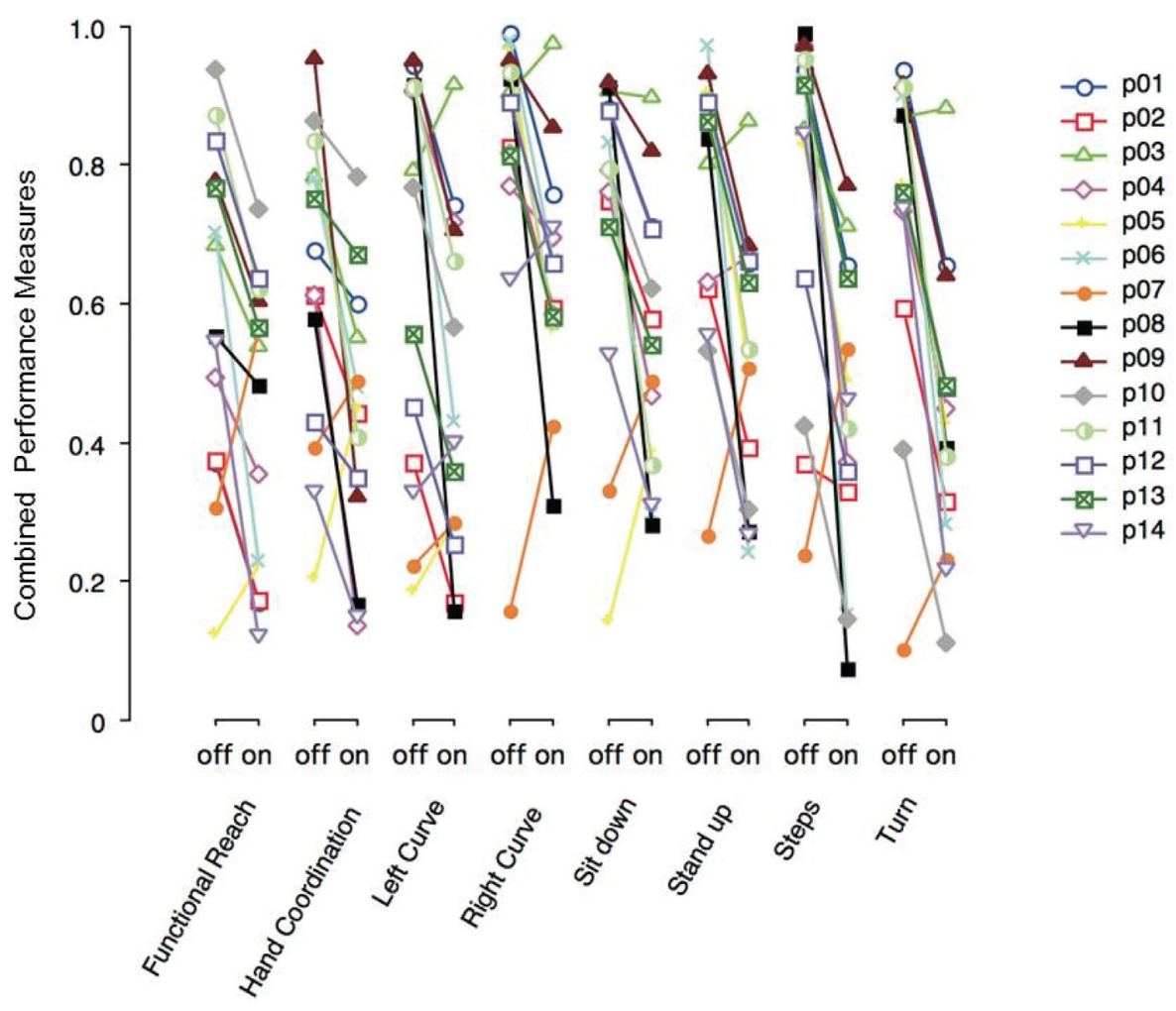

FIGURE 3 | Combined performance measures of Parkinson's disease (PD) patients with deep brain stimulation (DBS) switched-off or -on, separated by task. Each PD patient (p01-p14) is represented by a particular symbol and color.

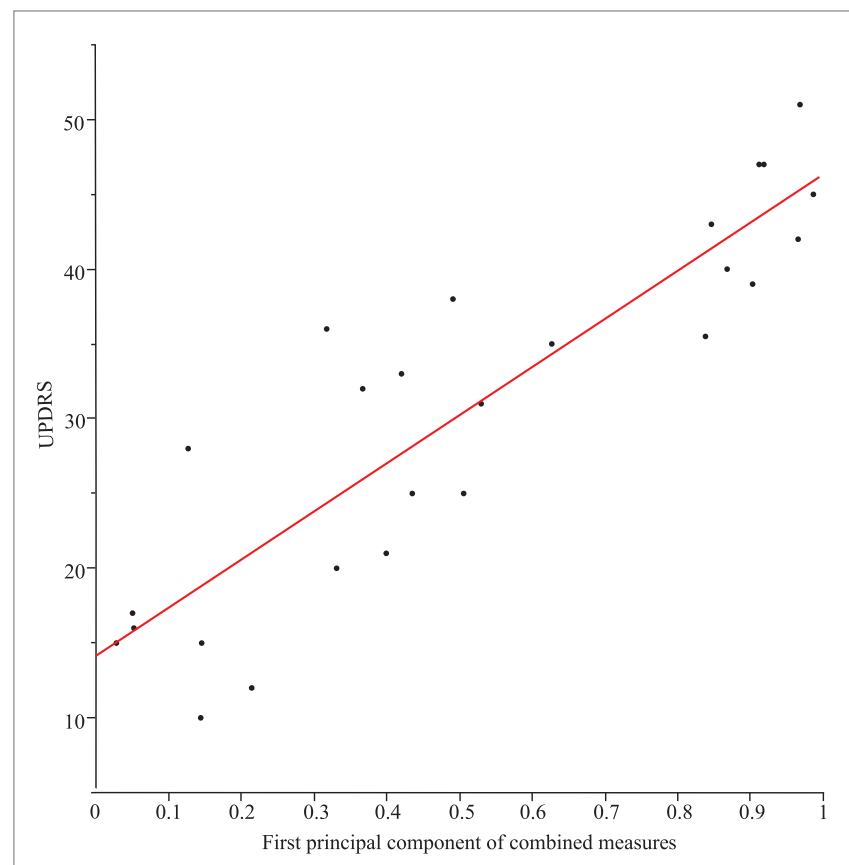

FIGURE 4 | Correlation between Unified Parkinson's Disease Rating Scale (UPDRS) (part III) and the first principal component of the combined measures from the different tasks $\left(r^{2}=0.79\right)$ for Parkinson's disease patients with DBS switched-off and switched-on. small or too large movement amplitudes, velocities, or ranges with respect to the normal subjects. Using many velocity traces from different joints, JA-related findings could also cover abnormal multisegmental control strategies in comparison to healthy subjects (46).

As a single velocity trace could remain within the range of normal behavior and still be abnormal due to high-frequency oscillations, we included additional motor-performance metrics associated with smoothness (e.g., NMAJ) or frequency content (i.e., Spectral Arc Length, SAL) of movement trajectories. Smoothness measures are known to be specifically abnormal in PD patients (14). Moreover, due to diverse calculation properties and normalization procedures, the smoothness measures used here characterize different traits of motor behavior.

Concerning the identification of a single metric that optimally distinguishes between normal and abnormal behavior, JA proved to be most successful in characterizing those motor tasks that involved whole-body movements (stand-up, turn, sit down, curve, and steps). In contrast, functional reach and hand coordination were characterized less precisely. On the other hand, there is evidence that the metric "Spectral Arc Length" covers abnormal arm movements in stroke patients (42). Here, SAL delivered high precision in PD patients' walking. However, other metrics outperformed it in all the other motor tasks. Interestingly, we found that "Speed Arc Length" was the most 
precise metric for many motor tasks (walking a $90^{\circ}$ curve, $180^{\circ}$ turning, hand coordination) while it was less precise for walking straight and functional reach. "Normalized Mean Absolute Jerk" outperformed other metrics for functional reach. The metric with large content of velocity and displacement, namely "Root Mean Square Jerk," revealed high precision for standing up and turning.

With the help of Random Forests, we sought optimal combinations of metrics to correctly classify PD patients off DBS and healthy subjects for each motor task separately. We used the accuracy of these metrics to compare the classification power of different metrics. Accuracy values were calculated using a leaveone-subject-out cross-validation. This procedure was repeated for each subject, and the values were averaged, resulting in a given Random Forest's average accuracy. In general, we observed that motor abnormalities in PD patients off DBS can be very precisely characterized via a set of metrics. The highest accuracy (94.6\%) was achieved for standing up. This corresponded to a sensitivity of $91.5 \%$ to detect a PD patient off DBS, and a specificity of $97.2 \%$ representing the rate of correctly identified healthy subjects. Thus, when testing the metrics to identify the one that optimally characterizes pathological behavior, we detected differences between tasks.

We then used the new metrics generated by Random Forests, which were derived from the classification results, to generate performance measures based on the mean of many recognition decisions between "zero" and "one" across the 161 metrics. They are related to the sum of probability distributions over the nodes of the decision trees. The purpose of this performance measure was to generate a measure for symptom severity. When applying the performance measure on PD patients on DBS, we identified a symptom severity of $53.3 \%$, as expected.

Since the optimal metric for measuring symptom severity in each motor task is different, one single new metric does not lead to high correlations across tasks. The low correlations between single metrics could have two potential sources. First, PD patients might exhibit individual combinations of deficits. Second, one single measure may be unsuitable for every motor task. To resolve this problem, we compared correlations between single metrics across tasks with correlations of task-specific Random Forests. The fact that the task-specific Random Forests correlated significantly better than any single measure indicates that the severities of PD patients' individual motor abnormalities in different motor tasks are highly interdependent. In other words, PD patients do not reveal a broadly scattered symptom severity across different tasks. This includes balance, fine motor skills, trunk movements, walking, and turning.

We suggest that PD patients' severity of motor symptoms is one-dimensional, i.e., they suffer from a common underlying motor deficit. This underlying motor deficit is an abstract measure, related to the first principal component across all motor abnormalities. It is related, e.g., to the slowness of standing up, smoothness of a fine motor skill of the hand, step length when walking. It is not a sum across all motor abnormalities, but the severity of motor abnormality in each motor task separately. This common motor deficit seems to affect different motor tasks in different ways. Consequently, the way how these motor abnormalities are optimally assessed, differs between tasks. With the help of task-specific extended Random Forests, we are capable of successfully deriving the common motor deficit no matter which task the PD patient is currently performing.

We plan to apply this common motor deficit measure to optimize therapeutic interventions, e.g., to adjust parameters for DBS electrodes (electrode location, amplitude, frequency, pulse width, direction of field). For a more automated assessment of PD patients' current motor state, we aim to complement the approach presented here by algorithms able to recognize the ongoing motor task in order to select the correct task-specific extended Random Forest.

\section{ETHICS STATEMENT}

All subjects and patients gave their written informed consent and their data were included anonymized according to the Declaration of Helsinki. We carried out this study in accordance with the guidelines of the Ethics Committee of the Medical Center, University of Freiburg and national guidelines. The Ethics Committee of the Medical Center, University of Freiburg approved the study protocol.

\section{AUTHOR CONTRIBUTIONS}

$\mathrm{AK}$ and CM contributed to the concept and design of the work, analysis and interpretation of data, and drafted the manuscript. $\mathrm{AK}$ was also responsible for data acquisition. TS and MC contributed to conceiving and designing the work, to acquiring data, and to critically revising the manuscript. IW drafted and edited the final manuscript for submission and revised the work critically. VC, WB, and CW contributed to the concept and critical revision of the work. All authors approved the final manuscript.

\section{ACKNOWLEDGMENTS}

The authors thank our Parkinson's disease patients for their valuable contribution to this study.

\section{FUNDING}

AK, TS, MC, IW, VC, WB, and CM were partially funded by the Brainlinks-Braintools Cluster of Excellence funded by the German Research Foundation (DFG, grant no ADV139). CM was funded by DFG (MA 2543/3-1).

\section{SUPPLEMENTARY MATERIAL}

The Supplementary Material for this article can be found online at http://www.frontiersin.org/article/10.3389/fneur.2017.00607/ full\#supplementary-material. 


\section{REFERENCES}

1. Goetz CG, Tilley BC, Shaftman SR, Stebbins GT, Fahn S, Martinez-Martin P, et al. Movement Disorder Society-sponsored revision of the Unified Parkinson's Disease Rating Scale (MDS-UPDRS): scale presentation and clinimetric testing results. Mov Disord (2008) 23(15):2129-70. doi:10.1002/ mds. 22340

2. Maetzler W, Klucken J, Horne M. A clinical view on the development of technology-based tools in managing Parkinson's disease. Mov Disord (2016) 31(9):1263-71. doi:10.1002/mds.26673

3. Bonora G, Mancini M, Carpinella I, Chiari L, Horak FB, Ferrarin M. Gait initiation is impaired in subjects with Parkinson's disease in the OFF state: evidence from the analysis of the anticipatory postural adjustments through wearable inertial sensors. Gait Posture (2017) 51:218-21. doi:10.1016/j. gaitpost.2016.10.017

4. Bonora G, Mancini M, Carpinella I, Chiari L, Ferrarin M, Nutt JG, et al. Investigation of anticipatory postural adjustments during one-leg stance using inertial sensors: evidence from subjects with Parkinsonism. Front Neurol (2017) 8:361. doi:10.3389/fneur.2017.00361

5. Cancela J, Pansera M, Pastorino M, Pastor L, Arredondo MT. Automatic assessment of bradykinesia severity in patients with Parkinson's disease. Paper Presented at: 7th International Conference on Wearable Micro and Nano Technologies for Personalized Health. Berlin, Germany (2010).

6. Pastorino M, Cancela J, Arredondo MT, Pastor-Sanz L, Contardi S, Valzania F. Preliminary results of ON/OFF detection using an integrated system for Parkinson's disease monitoring. Conf Proc IEEE Eng Med Biol Soc (2013) 2013:941-4. doi:10.1109/EMBC.2013.6609657

7. Griffiths RI, Kotschet K, Arfon S, Xu ZM, Johnson W, Drago J, et al. Automated assessment of bradykinesia and dyskinesia in Parkinson's disease. J Parkinsons Dis (2012) 2(1):47-55. doi:10.3233/JPD-2012-11071

8. Horne MK, McGregor S, Bergquist F. An objective fluctuation score for Parkinson's disease. PLoS One (2015) 10(4):e0124522. doi:10.1371/journal. pone. 0124522

9. Mera TO, Heldman DA, Espay AJ, Payne M, Giuffrida JP. Feasibility of homebased automated Parkinson's disease motor assessment. J Neurosci Methods (2012) 203(1):152-6. doi:10.1016/j.jneumeth.2011.09.019

10. Das S, Amoedo B, De la Torre F, Hodgins J. Detecting Parkinson's symptoms in uncontrolled home environments: a multiple instance learning approach. Conf Proc IEEE Eng Med Biol Soc (2012) 2012:3688-91. doi:10.1109/ EMBC.2012.6346767

11. Ferreira JJ, Godinho C, Santos AT, Domingos J, Abreu D, Lobo R, et al. Quantitative home-based assessment of Parkinson's symptoms: the SENSE-PARK feasibility and usability study. BMC Neurol (2015) 15:89. doi:10.1186/s12883-015-0343-Z

12. Hammerla NY, Fisher JM, Andras P, Rochester L, Walker R, Ploetz T. PD disease state assessment in naturalistic environments using deep learning. Paper presented at: Twenty-Ninth AAAI Conference on Artificial Intelligence. Austin, TX (2015)

13. Tzallas AT, Tsipouras MG, Rigas G, Tsalikakis DG, Karvounis EC, Chondrogiorgi M, et al. PERFORM: a system for monitoring, assessment and management of patients with Parkinson's disease. Sensors (Basel) (2014) 14(11):21329-57. doi:10.3390/s141121329

14. Del Din S, Godfrey A, Galna B, Lord S, Rochester L. Free-living gait characteristics in ageing and Parkinson's disease: impact of environment and ambulatory bout length. J Neuroeng Rehabil (2016) 13(1):46. doi:10.1186/ s12984-016-0154-5

15. Dinov ID, Heavner B, Tang M, Glusman G, Chard K, Darcy M, et al. Predictive big data analytics: a study of Parkinson's disease using large, complex, heterogeneous, incongruent, multi-source and incomplete observations. PLoS One (2016) 11(8):e0157077. doi:10.1371/journal.pone. 0157077

16. Joshi D, Khajuria A, Joshi P. An automatic non-invasive method for Parkinson's disease classification. Comput Methods Programs Biomed (2017) 145:135-45. doi:10.1016/j.cmpb.2017.04.007

17. Klucken J, Barth J, Kugler P, Schlachetzki J, Henze T, Marxreiter F, et al. Unbiased and mobile gait analysis detects motor impairment in Parkinson's disease. PLoS One (2013) 8(2):e56956. doi:10.1371/journal.pone. 0056956
18. Chaturvedi M, Hatz F, Gschwandtner U, Bogaarts JG, Meyer A, Fuhr P, et al. Quantitative EEG (QEEG) measures differentiate Parkinson's Disease (PD) patients from healthy controls (HC). Front Aging Neurosci (2017) 9:3. doi:10.3389/fnagi.2017.00003

19. Pereira CR, Pereira DR, Silva FA, Masieiro JP, Weber SAT, Hook C, et al. A new computer vision-based approach to aid the diagnosis of Parkinson's disease. Comput Methods Programs Biomed (2016) 136:79-88. doi:10.1016/j. cmpb.2016.08.005

20. Bernad-Elazari H, Herman T, Mirelman A, Gazit E, Giladi N, Hausdorff JM. Objective characterization of daily living transitions in patients with Parkinson's disease using a single body-fixed sensor. JNeurol (2016) 263(8):1544-51. doi:10.1007/s00415-016-8164-6

21. Martinez-Manzanera O, Roosma E, Beudel M, Borgemeester RWK, van Laar T, Maurits NM. A method for automatic and objective scoring of bradykinesia using orientation sensors and classification algorithms. IEEE Trans Biomed Eng (2016) 63(5):1016-24. doi:10.1109/TBME.2015. 2480242

22. Roy SH, Cole BT, Gilmore LD, De Luca CJ, Thomas CA, Saint-Hilaire MM, et al. High-resolution tracking of motor disorders in Parkinson's disease during unconstrained activity. Mov Disord (2013) 28(8):1080-7. doi:10.1002/ mds. 25391

23. Henderson EJ, Lord SR, Close JCT, Lawrence AD, Whone A, BenShlomo Y. The ReSPonD trial - rivastigmine to stabilise gait in Parkinson's disease a phase II, randomised, double blind, placebo controlled trial to evaluate the effect of rivastigmine on gait in patients with Parkinson's disease who have fallen. BMC Neurol (2013) 13:188. doi:10.1186/1471-237713-188

24. Markle-Reid M, Browne G, Gafni A, Roberts J, Weir R, Thabane L, et al. A cross-sectional study of the prevalence, correlates, and costs of falls in older home care clients "at risk" for falling. Can J Aging (2010) 29(1):119-37. doi:10.1017/S0714980809990365

25. Godfrey A, Bourke A, Del Din S, Morris R, Hickey A, Helbostad JL, et al. Towards holistic free-living assessment in Parkinson's disease: unification of gait and fall algorithms with a single accelerometer. Conf Proc IEEE Eng Med Biol Soc (2016) 2016:651-4. doi:10.1109/EMBC.2016.7590786

26. Silva de Lima AL, Evers LJW, Hahn T, Bataille L, Hamilton JL, Little MA, et al. Freezing of gait and fall detection in Parkinson's disease using wearable sensors: a systematic review. J Neurol (2017) 264(8):1642-54. doi:10.1007/ s00415-017-8424-0

27. Lord S, Godfrey A, Galna B, Mhiripiri D, Burn D, Rochester L. Ambulatory activity in incident Parkinson's: more than meets the eye? J Neurol (2013) 260(12):2964-72. doi:10.1007/s00415-013-7163-0

28. Lord S, Galna B, Rochester L. Moving forward on gait measurement: toward a more refined approach. Mov Disord (2013) 28(11):1534-43. doi:10.1002/ mds. 25545

29. Mollenhauer B, Rochester L, Chen-Plotkin A, Brooks D. What can biomarkers tell us about cognition in Parkinson's disease? Mov Disord (2014) 29(5):622-33. doi: $10.1002 / \mathrm{mds} .25846$

30. Esser P, Dawes H, Collett J, Feltham MG, Howells K. Assessment of spatiotemporal gait parameters using inertial measurement units in neurological populations. Gait Posture (2011) 34(4):558-60. doi:10.1016/j.gaitpost.2011. 06.018

31. Esser P, Dawes H, Collett J, Feltham MG, Howells K. Validity and inter-rater reliability of inertial gait measurements in Parkinson's disease: a pilot study. J Neurosci Methods (2012) 205(1):177-81. doi:10.1016/j.jneumeth.2012. 01.005

32. Salarian A, Russmann H, Vingerhoets FJG, Dehollain C, Blanc Y, Burkhard PR, et al. Gait assessment in Parkinson's disease: toward an ambulatory system for long-term monitoring. IEEE Trans Biomed Eng (2004) 51(8):1434-43. doi:10.1109/TBME.2004.827933

33. Trojaniello D, Cereatti A, Pelosin E, Avanzino L, Mirelman A, Hausdorff JM, et al. Estimation of step-by-step spatio-temporal parameters of normal and impaired gait using shank-mounted magneto-inertial sensors: application to elderly, hemiparetic, parkinsonian and choreic gait. J Neuroeng Rehabil (2014) 11:152. doi:10.1186/1743-0003-11-152

34. Trojaniello D, Ravaschio A, Hausdorff JM, Cereatti A. Comparative assessment of different methods for the estimation of gait temporal parameters using a single inertial sensor: application to elderly, post-stroke, Parkinson's 
disease and Huntington's disease subjects. Gait Posture (2015) 42(3):310-6. doi:10.1016/j.gaitpost.2015.06.008

35. Mancini M, El-Gohary M, Pearson S, McNames J, Schlueter H, Nutt JG, et al. Continuous monitoring of turning in Parkinson's disease: rehabilitation potential. NeuroRehabilitation (2015) 37(1):3-10. doi:10.3233/ NRE-151236

36. Smith E, Walsh L, Doyle J, Greene B, Blake C. The reliability of the quantitative timed up and go test (QTUG) measured over five consecutive days under single and dual-task conditions in community dwelling older adults. Gait Posture (2016) 43:239-44. doi:10.1016/j.gaitpost.2015.10.004

37. Zampieri C, Salarian A, Carlson-Kuhta P, Nutt JG, Horak FB. Assessing mobility at home in people with early Parkinson's disease using an instrumented Timed Up and Go test. Parkinsonism Relat Disord (2011) 17(4): 277-80. doi:10.1016/j.parkreldis.2010.08.001

38. El-Gohary M, Pearson S, McNames J, Mancini M, Horak F, Mellone S, et al. Continuous monitoring of turning in patients with movement disability. Sensors (Basel) (2013) 14(1):356-69. doi:10.3390/s140100356

39. Bloem BR, Marinus J, Almeida Q, Dibble L, Nieuwboer A, Post B, et al. Measurement instruments to assess posture, gait, and balance in Parkinson's disease: critique and recommendations. Mov Disord (2016) 31(9):1342-55. doi:10.1002/mds.26572

40. Tomlinson CL, Stowe R, Patel S, Rick C, Gray R, Clarke CE. Systematic review of levodopa dose equivalency reporting in Parkinson's disease. Mov Disord (2010) 25(15):2649-53. doi:10.1002/mds.23429

41. Kuhner A, Schubert T, Cenciarini M, Maurer C, Burgard W. A probabilistic approach based on Random forests to estimating similarity of human motion in the context of Parkinson's Disease. 2016 IEEE/RSJ International Conference on Intelligent Robots and Systems (IROS). Daejeon (2016). p. 1851-6.
42. Balasubramanian S, Melendez-Calderon A, Burdet E. A robust and sensitive metric for quantifying movement smoothness. IEEE Trans Biomed Eng (2012) 59(8):2126-36. doi:10.1109/TBME.2011.2179545

43. Breiman L. Random forests. Mach Learn (2001) 45(1):5-32. doi:10.1023/ A:1010933404324

44. Wouda FJ, Giuberti M, Bellusci G, Veltink PH. Estimation of full-body poses using only five inertial sensors: an eager or lazy learning approach? Sensors (Basel) (2016) 16(12):E2138. doi:10.3390/s16122138

45. Semwal SK, Hightower R, Stansfield S. Mapping algorithms for realtime control of an avatar using eight sensors. Presence (1998) 7(1):1-21. doi:10.1162/105474698565497

46. Burget F, Maurer C, Burgard W, Bennewitz M. Learning motor control parameters for motion strategy analysis of Parkinson's disease patients. 2015 IEEE/RSJ International Conference on Intelligent Robots and Systems (IROS). Hamburg (2015). p. 5019-25.

Conflict of Interest Statement: The authors declare that the research was conducted in the absence of any commercial or financial relationships that could be construed as a potential conflict of interest.

Copyright (c) 2017 Kuhner, Schubert, Cenciarini, Wiesmeier, Coenen, Burgard, Weiller and Maurer. This is an open-access article distributed under the terms of the Creative Commons Attribution License (CC BY). The use, distribution or reproduction in other forums is permitted, provided the original author(s) or licensor are credited and that the original publication in this journal is cited, in accordance with accepted academic practice. No use, distribution or reproduction is permitted which does not comply with these terms. 